\title{
Manejo perioperatorio del paciente anticoagulado con fibrilación auricular no valvular
}

\author{
Dras. Sofía Noria', Florencia Di Landro
}

\begin{abstract}
Resumen el manejo más adecuado de estos fármacos.

Palabras clave: $\quad$ FIBRILACIÓN AURICULAR NO VALVULAR

ANTICOAGULANTES

PERÍODO PERIOPERATORIO
\end{abstract}

La fibrilación auricular (FA) aumenta el riesgo de accidente cerebrovascular (ACV) y tromboembolia (TE) sistémica, por lo que resulta fundamental prevenir esta temible complicación a través del tratamiento anticoagulante. Al uso habitual de warfarina se agregaron en los últimos años los anticoagulantes orales directos (ACOD).

Frente a una intervención, o procedimiento invasivo es necesario evaluar el riesgo embólico y el de sangrado para definir

En el presente artículo se proponen recomendaciones para el tratamiento anticoagulante periprocedimiento en la fibrilación auricular no valvular basadas en el consenso de expertos del American College of Cardiology.

\section{Perioperative management of the anticoagulated patient with non-valvular atrial fibrillation}

\section{Summary}

Atrial fibrillation increases the risk of stroke and systemic embolism, so it is important to prevent this terrible complication with anticoagulant therapy. To the usual use of warfarin were added in recent years, direct anticoagulants.

In front of an invasive procedure, it is necessary to evaluate embolism and bleeding risks to define the most appropriate management of these drugs.

In the present article, recommendations based in expert consensus of American College of Cardiology are proposed, to periprocedural anticoagulation treatment in non-valvular atrial fibrillation.

Key words: $\quad$ NON-VALVULAR ATRIAL FIBRILLATION

ANTICOAGULANTS

PERIOPERATIVE PERIOD

\section{Introducción}

Cada vez es más frecuente en nuestra práctica clínica la evaluación de pacientes anticoagulados por FA no valvular (FANV) que serán sometidos a un procedimiento invasivo.

Frente a cada paciente es necesario considerar el riesgo TE versus el riesgo de sangrado periprocedimiento de manera de determinar la mejor estrategia a seguir.

La evidencia al respecto es limitada, sobre todo con el manejo de los anticoagulantes orales de ac- ción directa (ACOD), siendo la mayoría de las recomendaciones, opinión de expertos.

En el presente artículo se realiza una revisión de las evidencias sobre el uso de anticoagulantes orales (ACO) en pacientes con FANV que serán sometidos a procedimientos invasivos, usando como referencia el consenso de expertos del American College of Cardiology (ACC) 2017(1).

\section{Importancia del tema}

La FA es la arritmia sostenida más frecuente en el 


\begin{tabular}{|c|c|c|c|}
\hline $\begin{array}{l}\text { Sangrado clínicamente no } \\
\text { importante }\end{array}$ & $\begin{array}{l}\text { Intervenciones con bajo riesgo de } \\
\text { sangrado }\end{array}$ & $\begin{array}{l}\text { Intervenciones con riesgo de } \\
\text { sangrado intermedio }\end{array}$ & $\begin{array}{l}\text { Intervenciones con riesgo de } \\
\text { sangrado alto }\end{array}$ \\
\hline Cirugía de cataratas & $\begin{array}{l}\text { Ablación TSV, flutter } \\
\text { auricular y mayoría de FA }\end{array}$ & $\begin{array}{l}\text { Extracción }>3 \text { piezas } \\
\text { dentales }\end{array}$ & Ablación TV epicárdica \\
\hline \multirow{6}{*}{$\begin{array}{l}\text { Tratamiento de } \\
\text { retinopatía, } \\
\text { fotocoagulación }\end{array}$} & $\begin{array}{l}\text { Implante de marcapasos } \\
\text { y CDI }\end{array}$ & Biopsia pleural & Cirugía espinal \\
\hline & Blefaroplastia & Histerectomía & Cirugía ortopédica mayor \\
\hline & $\begin{array}{l}\text { Extracción de 1-3 piezas } \\
\text { dentales }\end{array}$ & & Punción lumbar \\
\hline & CACG por acceso radial & & Biopsia renal \\
\hline & $\begin{array}{l}\text { Endoscopía digestiva con } \\
\text { biopsia }\end{array}$ & & $\begin{array}{l}\text { Resección prostática } \\
\text { transuretral }\end{array}$ \\
\hline & & & $\begin{array}{l}\text { Cirugía aorta abdominal y } \\
\text { torácica }\end{array}$ \\
\hline
\end{tabular}

TSV: taquicardia supraventricular; CDI: cardiodesfibrilador implantable; CACG: angiografía coronaria; TV: taquicardia ventricular.

mundo, su prevalencia aumenta con la edad y uno de cada cuatro individuos la padecerá durante su vida ${ }^{(1)}$. La terapia antitrombótica está recomendada para la mayoría de los pacientes con FA para reducir el riesgo de ACV, TE sistémica y mortalidad. Dado que el riesgo anual de ACV es inhomogéneo(2), se recomienda calcular el riesgo TE utilizando el score $\mathrm{CHA}_{2} \mathrm{DS}_{2}$ VASc e iniciar la anticoagulación oral con valores $\geq 2$. Más del $85 \%$ de los pacientes con FA tienen un score $\geq 2$ requiriendo, por lo tanto, anticoagulación crónica ${ }^{(3)}$.

Definimos FANV como aquella que no está vinculada a estenosis mitral reumática, prótesis valvular mecánica o biológica o reparación valvular mitral ${ }^{(1)}$.

La interrupción temporaria (IT), o la omisión de una o más dosis de un ACO, son necesarias a menudo, para disminuir el riesgo de sangrados frente a procedimientos quirúrgicos o invasivos. Aunque varios factores son considerados cuando se toma la decisión de interrumpir la anticoagulación, la práctica varía ampliamente ${ }^{(1)}$.

Como terapia puente (TP) se entiende el proceso mediante el cual un ACO es interrumpido y sustituido por un anticoagulante subcutáneo o intravenoso, antes o después de un procedimiento invasivo ${ }^{(1)}$.

Se abordarán los siguientes aspectos: 1) cuándo y en qué casos debe interrumpirse la terapia anticoagulante; 2 ) cuándo y cómo debe realizarse la TP con un anticoagulante por vía parenteral, y 3) cuándo y cómo debe reiniciarse la terapia anticoagulante para aquellos que requieran IT.
1. Cuándo y en qué casos debe interrumpirse la terapia anticoagulante

El esquema que se analizará es utilizado para procedimientos planificados electivos, no para aquellos que ocurren de urgencia o emergencia.

Antes de que se pueda determinar si la IT es necesaria para la realización de un procedimiento, es importante considerar: 1) el riesgo de sangrado del procedimiento; 2) el efecto clínico del sangrado en caso de que ocurra; 3 ) qué factores del paciente pueden aumentar el riesgo de sangrado ${ }^{(1)}$.

\subsection{Valoración del riesgo de sangrado del procedimiento y de su efecto clínico}

La decisión de suspender o continuar con la anticoagulación frente a un procedimiento invasivo está en relación con el riesgo hemorrágico estimado. Tan importantes como la prevalencia de sangrados, son sus consecuencias. Por ejemplo, un sangrado aun de escasa magnitud durante la anestesia neuroaxial, o después de cirugía cardíaca, intraocular, intracraneal o espinal puede resultar en morbilidad o mortalidad significativas. Por lo tanto, procedimientos con bajas tasas de sangrado, pero secuelas significativas asociadas, deben ser categorizados como de alto riesgo ${ }^{(1)}$.

La clasificación propuesta por el ACC 2017, que se utilizará como referencia, realizada con el aporte de varias sociedades profesionales, estratifica los procedimientos más frecuentes en cuatro niveles de riesgo de sangrado:clínicamente no importante, bajo riesgo o riesgo intermedio y alto ${ }^{(1)}$ (tabla 1$)$. 


\subsection{Valoración de riesgo de sangrado del paciente}

Más allá del riesgo de sangrado inherente a un procedimiento determinado, también es importante valorar los factores relacionados con el paciente que puedan incrementar el mismo. Estos incluyen: historia de sangrado previo (particularmente en los tres meses previos), sangrado con un procedimiento similar o con TP previa, anormalidad de la función plaquetaria cuali o cuantitativa, uso concomitante de terapia antiplaquetaria (u otra medicación/suplemento asociado con disfunción plaquetaria) y uso de inhibidor de vitamina $K$ (AVK) con valor de INR superior al rango terapéutico. Si es posible, se debe retrasar el procedimiento para que los factores relacionados con el paciente puedan ser corregi$\operatorname{dos}^{(1)}$.

Varios scores fueron propuestos para evaluar el riesgo de sangrado en pacientes con FA. El más utilizado es el HAS-BLED, que considera: hipertensión, disfunción hepática o renal, accidente isquémico transitorio (AIT) o ACV previos, historia de sangrado mayor, INR lábil, edad mayor a 65 años, $\mathrm{e}$ interacción con drogas ${ }^{(1)}$.

Aunque el score HAS-BLED ha demostrado tener un valor predictivo periprocedimiento, no está específicamente respaldado por las actuales guías para este propósito(1).

\section{Pacientes bajo tratamiento con inhibidores de vitamina $\mathrm{K}$}

La warfarina es el AVK más utilizado en el mundo. Tiene una vida media de 36 a 42 horas, lo que requiere una planificación de IT de ser necesaria. Se propone el siguiente esquema de manejo periprocedimiento $^{(1)}$.

Guía para determinar si un inhibidor de vitamina $K$ debe ser interrumpido periprocedimiento

a) No interrumpir terapia con AVK en pacientes que van a someterse a procedimientos:

- con bajo riesgo de sangrado o no significativo;

- ausencia de factores propios que aumenten el riesgo de sangrado.

b) Interrumpir terapia con AVK en:

- pacientes que serán sometidos a procedimientos de intermedio o alto riesgo;

- pacientes que se realizarán procedimientos con riesgo de sangrado incierto y/o con factores que aumentan el riesgo de sangrado.

c) Considerar la posibilidad de interrumpir un AVK sobre la base del juicio clínico en pacientes que se someten a procedimientos con:

- riesgo de sangrado no significativo o bajo pero con factores que aumentan este riesgo;
- riesgo de sangrado incierto y ausencia de factores que aumenten este riesgo.

Para todos los pacientes que reciben AVK se debe obtener un INR cinco a siete días antes del procedimiento. Esto se realiza en individuos que no requieren IT para identificar a los que presenten un INR $>3$ o en los que sí requieren IT para determinar el número de días que el AVK debe ser suspendido.

Interrupción de inhibidores de vitamina $\mathrm{K}$ periprocedimiento

a) En aquellos pacientes con INR de 1,5-1,9, el AVK debe ser discontinuado tres o cuatro días previos al procedimiento si se desea llegar a un INR normal o por un período más corto si un INR mayor pero subterapéutico es aceptable.

b) Cuando el INR es 2-3, el AVK debe ser discontinuado cinco días previos al procedimiento.

c) Si el INR es $>3$, el AVK debe ser discontinuado al menos cinco días previos al procedimiento. La duración exacta va a depender del INR actualizado, del tiempo hasta el procedimiento programado y del INR deseado.

d) En los que reciben una dosis alta de AVK de mantenimiento (7,5 a $10 \mathrm{mg}$ /día o mayor) o en aquellos cuyo INR se normalice más rápido, una interrupción más breve puede ser suficiente previo al procedimiento.

En todos los casos, el INR debe ser revisado 24 horas antes del procedimiento, particularmente si se desea llegar a un INR normal. Si persiste elevado, el procedimiento debe posponerse de ser posible hasta que se alcance un valor adecuado.

Pacientes que reciben anticoagulantes orales directos

Actualmente están aprobados cuatro ACOD para reducir el riesgo de ACV o embolia sistémica en la FANV: apixabán, edoxabán, rivaroxabán y dabigatrán ${ }^{(1)}$. Tienen una ventana terapéutica amplia, efecto anticoagulante predecible, inicio y final de acción rápidos y no requieren control rutinario de los niveles de anticoagulación ${ }^{(4)}$. Varían en su farmacocinética, frecuencia de dosificación, dependencia de excreción renal, y criterios para ajustar sus dosis. Su vida media relativamente corta suele reducir el intervalo de suspensión (en comparación con AVK) de la anticoagulación periprocedimiento ${ }^{(1)}$.

Es importante tener en cuenta la farmacocinética de los ACOD. Debido a la variación entre el pico y los niveles de droga durante el intervalo de dosis con toma regular una o dos veces al día, es posible realizar el procedimiento durante el final de un in- 


\begin{tabular}{|c|c|c|c|c|}
\hline $\mathrm{ClCr}$ & Dabigatrán & Dabigatrán & $\begin{array}{c}\text { Apixabán-Edoxabán- } \\
\text { Rivaroxabán }\end{array}$ & $\begin{array}{c}\text { Apixabán-Edoxabán- } \\
\text { Rivaroxabán }\end{array}$ \\
\hline & Bajo riesgo & $\begin{array}{c}\text { Incierto, intermedio } \\
\text { o alto }\end{array}$ & Bajo riesgo & Alto riesgo \\
\hline$\geq 80 \mathrm{ml} / \mathrm{min}$ & $\geq 24 \mathrm{~h}$ & $\geq 48 \mathrm{~h}$ & $\geq 24 \mathrm{~h}$ & $\geq 48 \mathrm{~h}$ \\
\hline $50-79 \mathrm{ml} / \mathrm{min}$ & $\geq 36 \mathrm{~h}$ & $\geq 72 \mathrm{~h}$ & $\geq 24 \mathrm{~h}$ & $\geq 48 \mathrm{~h}$ \\
\hline $30-49 \mathrm{ml} / \mathrm{min}$ & $\geq 48 \mathrm{~h}$ & $\geq 96 \mathrm{~h}$ & $\geq 24 \mathrm{~h}$ & $\geq 48 \mathrm{~h}$ \\
\hline $15-29 \mathrm{ml} / \mathrm{min}$ & $\geq 72 \mathrm{~h}$ & $\geq 120 \mathrm{~h}$ & $\geq 36 \mathrm{~h}$ & $\begin{array}{l}\text { No hay datos, } \\
\text { considerar } \geq 72 \mathrm{~h}\end{array}$ \\
\hline$<15 \mathrm{ml} / \mathrm{min}$ & $\begin{array}{l}\text { No hay datos, } \\
\text { considerar } \geq 96 \mathrm{~h}\end{array}$ & No hay datos & $\begin{array}{l}\text { No hay datos, } \\
\text { considerar } \geq 48 \mathrm{~h}\end{array}$ & \\
\hline
\end{tabular}

tervalo de dosis y reiniciar el ACOD en la noche o el día después con omisión de una sola dosis o en algunos casos sin pérdida de dosis ${ }^{(1)}$.

Desde que los ACOD están disponibles, una preocupación frecuente ha sido la falta de antídoto específico en el caso de un sangrado mayor. Esto es particularmente relevante en el ajuste periprocedimiento y en pacientes que requieren procedimientos repetidos. Recientemente se han producido avances en esta área con la aprobación del anticuerpo monoclonal idarucizumab para revertir la acción de dabigatrán y andexanet para contrarrestar los efectos anticoagulantes de rivaroxabán y apixabán ${ }^{(1)}$.

Salvo que la situación sea considerada de riesgo hemorrágico mínimo, en cuyo caso no se requiere la suspensión de ACOD (únicamente se omitirá la toma coincidente con el procedimiento), el tratamiento se suspenderá con una antelación que dependerá del riesgo hemorrágico, del agente específico y del tiempo necesario para que se produzca pérdida del efecto anticoagulante (lo cual estará en relación, a su vez, con el aclaramiento de creatinina $)^{(1,5)}$.

Para los pacientes bajo ACOD que necesitan IT es importante valorar la función renal para determinar la duración del efecto anticoagulante una vez que el agente es discontinuado (cuatro o cinco vidas medias). Esto debe ser realizado en función de la ecuación de Cockcroft-Gault (con el peso actual) para calcular el clearance de creatinina $(\mathrm{Cl} \mathrm{Cr})^{(1)}($ tabla 2$)$.

Se recomienda iniciar con la valoración de los factores que aumenten el riesgo de sangrado relacionados con el paciente. Esto se debe en parte a la escasez de datos acerca de qué procedimientos se pueden realizar de forma segura en pacientes que reciben ACOD sin realizar $I T^{(1)}$.

La duración recomendada de la IT para cada
ACOD depende del $\mathrm{Cl} \mathrm{Cr} /$ metabolismo esperado del fármaco, del riesgo de sangrado del procedimiento, y de los factores relacionados con el paciente que también aumenten el riesgo. En los pacientes con alto riesgo de sangrado los procedimientos coordinados deben ser retrasados, de ser posible, para corregir los factores de riesgo. Si el procedimiento no puede ser pospuesto o los factores relacionados con el paciente no pueden ser corregidos, el ACOD debe ser interrumpido según el juicio clínico. El uso de idarucizumab puede ser considerado en pacientes que reciben dabigatrán que van a procedimientos de urgencia/emergencia (menos de 8 horas) asociados con alto riesgo de sangrado que requieran una hemostasia normal (1).

En pacientes sin factores de riesgo es importante valorar el riesgo de sangrado del procedimiento. En aquellos que van a procedimientos sin riesgo importante, el ACOD solo debe ser suspendido por una dosis. Alternativamente, el procedimiento puede ser realizado sin IT, pero sincronizado, para coincidir con el nadir predicho del ACOD. Los procedimientos realizados con bajo riesgo de sangrado (por ejemplo, cirugía de cataratas) son posiblemente mejor realizados con interrupción limitada o sin interrupción, pero la experiencia con ACOD es escasa(1).

Considerando la vida media corta de los ACOD y su rápido inicio de acción, su período de suspensión ante un procedimiento invasivo es menor que el de los AVK. Teniendo en cuenta estas características farmacocinéticas, no se recomienda el uso de TP en caso de suspensión temporal de ACOD, salvo en aquellos pacientes en los que la toma oral tras el procedimiento no sea factible. Sin embargo, la seguridad de esta recomendación no ha sido suficientemente corroborada. A este respecto, se han publicado subestudios post hoc de los ensayos con ACOD, 
en los que se ha evaluado la presencia de complicaciones durante el período periprocedimiento. Los resultados demuestran que la incidencia de eventos hemorrágicos y de TE fue similar en los sujetos tratados con warfarina o ACOD. En dos de ellos se describen además los resultados de los pacientes que recibieron TP con heparina (decisión a criterio del investigador), y se observó que su administración, en los que suspendían el ACOD, aumentaba el número de hemorragias sin descenso significativo de los eventos $\mathrm{TE}^{(5)}$. Resultados similares se comunicaron en un subestudio del RE-LY en el cual los pacientes bajo tratamiento con dabigatrán o warfarina a los que se les suspendía dicha medicación debido a un procedimiento intervencionista, mostraron un aumento del riesgo de sangrado cuando se realizaba $\mathrm{TP}^{(6)}$

Uso de anticoagulantes orales directos en periprocedimientos neuroaxiales

El uso de anticoagulantes concomitante a la aplicación de anestesia neuroaxial aumenta el riesgo de producir hematomas espinales o epidurales, con consecuencias catastróficas. La Sociedad Americana de Anestesia Regional y Medicina del dolor recomienda discontinuar los ACOD durante cuatro a cinco días para dabigatrán y tres a cinco días para los inhibidores del factor Xa, reiniciándolos a las 24 horas posprocedimiento. Esta estrategia es razonable para los pacientes de bajo riesgo trombótico. En el caso de los de elevado riesgo trombótico, considerar un intervalo sin medicamentos de dos o tres vidas medias, o considerar la TP con heparinas de bajo peso molecular (HBPM) $)^{(1)}$.

2) Cuándo y cómo debe realizarse terapia puente con un anticoagulante por vía parenteral

En general, el riesgo TE asociado con la interrupción del tratamiento anticoagulante oral sinTP con heparina es bajo. Además, datos recientes demuestran que la TP con heparina se asocia con mayor riesgo de hemorragia sin beneficios en la incidencia de eventos $T E^{(7)}$. La mayoría de los estudios publicados para evaluar la seguridad de la interrupción de la anticoagulación periprocedimiento fueron realizados con AVK.

En el ensayo BRUISE CONTROL se incluyeron pacientes que iban a implante de marcapasos o DAI bajo tratamiento con un AVK que no fue interrumpido (INR 3, el día del procedimiento) en comparación con IT y puente con heparina, con un riesgo anual de eventos TE de 5\%. Los resultados obtenidos permitieron concluir que los que no interrum- pieron el tratamiento con warfarina, y por lo tanto no pasaron a TP con heparina, tuvieron reducción de la incidencia de hematoma en el lugar del implante, sin diferencias en otras variables (riesgo relativo 0,19; IC 95\% 0,10-0,36; $\mathrm{p}<0,001)^{(8)}$.

En una revisión, que incluyó 34 estudios sobre TP periprocedimiento en pacientes anticoagulados con AVK, se demostró que quienes la recibían presentaban un riesgo aumentado de sangrado total y sangrado mayor, con un riesgo similar de eventos $\mathrm{TE}$ en comparación con los pacientes sin TP. Si bien esta revisión incluye un gran número de pacientes, una importante limitación es la heterogeneidad en la interpretación de los eventos (TE y sangrados), sumado a que solo uno de los trabajos incluidos era aleatorizado y el resto eran observacionales, lo que no permite sacar conclusiones definitivas. De todas maneras, recomienda que en los pacientes con alto riesgo de sangrado y bajo riesgo $\mathrm{TE}$ no se realice $\mathrm{TP}^{(9)}$.

Por otra parte, los datos del registro ORBIT-AF de pacientes anticoagulados por FA tampoco apoyan el uso sistemático de TP, ya que aquellos pacientes que la recibieron, presentaron más riesgo de hemorragia mayor (OR 3,48; $\mathrm{p}<0,0001)$, de TE (OR $1,62 ; \mathrm{p}=0,07)$, de la combinación de eventos hemorrágicos, TE o muerte (OR 1,94; $\mathrm{p}=0,0001)^{(10)}$. No obstante, debido a las limitaciones metodológicas inherentes a un estudio observacional y al escaso número de pacientes con riesgo alto de TE, no se puede excluir que en este último grupo la TP pueda ser beneficiosa ${ }^{(5)}$.

El ensayo clínico BRIDGE comparó el uso de dalteparina con placebo en pacientes con FA en los que se interrumpió transitoriamente la anticoagulación con warfarina debido a un procedimiento invasivo. Se excluyeron los casos con alto riesgo TE, como los portadores de prótesis valvulares y ACV en los 12 meses previos. El objetivo primario fue valorar los eventos TE arteriales (ACV, embolia sistémica o AIT) y el sangrado mayor. Los resultados mostraron similar incidencia de eventos TE en ambos grupos (0,3\% en el grupo dalteparina frente a $0,4 \%$ en el grupo placebo; $\mathrm{p}=0,01$ para no inferioridad). En cuanto a los sangrados mayores fueron superiores en el grupo que recibió dalteparina ( 3,2 versus $1,3 \%$; $p=0,005$ para superioridad). Estos hallazgos demuestran que la no realización de TP fue no inferior para la prevención de TE y que la misma aumenta el riesgo de sangrado mayor ${ }^{(6)}$.

Los recientes resultados del estudio BRUISE CONTROL-2 no mostraron diferencias en la incidencia de eventos isquémicos o hemorrágicos entre mantener o interrumpir el tratamiento con $\operatorname{ACOD}^{(7)}$. 
Tabla 3. Estratificación del riesgo tromboembólico de los pacientes en tratamiento anticoagulante por fibrilación auricular. Modificada de ${ }^{(7)}$.

Riesgo

$\begin{array}{ll}\text { Alto ( }>10 \% \text { al año) } & \begin{array}{l}\mathrm{CHA}_{2} \mathrm{DS}_{2} \text {-VASc 7-9 } \\ \text { ACV/AIT }<3 \text { meses }\end{array} \\ \text { Moderado (5\% a 10\% al año) } & \begin{array}{l}\mathrm{CHA}_{2} \mathrm{DS}_{2}-\text { VASc } 5-6 \\ \text { ACV/AIT }>3 \text { meses }\end{array} \\ \text { Bajo (<5\% al año) } & \begin{array}{l}\mathrm{CHA}_{2} \mathrm{DS}_{2} \text {-VASc 1-4 } \\ \text { Sin ACV ni AIT previo }\end{array}\end{array}$

ACV: accidente cerebrovascular; AIT: accidente isquémico transitorio

Los resultados publicados refuerzan la recomendación de omitir la TP en pacientes con riesgo bajo de TE y no aportan evidencia suficiente en contra de de indicarla en los pacientes de riesgo alto. En caso de riesgo intermedio se podría considerar su uso en aquellos pacientes que requieran la suspensión de AVK durante un tiempo prolongado y cuyo riesgo hemorrágico sea bajo ${ }^{(5)}$.

\section{Guía para determinar si es necesario realizar terapia puente}

Es necesario realizar valoración del riesgo TE en pacientes bajo tratamiento anticoagulante (tabla $3)$.

- Pacientes de bajo riesgo: discontinuar el AVK antes del procedimiento y reanudar sin puente $^{(1)}$.

- Pacientes con riesgo moderado: determinar el riesgo de sangrado del paciente para determinar la conveniencia de la TP:

1. Si el riesgo de sangrado es alto, se recomienda la interrupción del AVK sin puente.

2. Si no hay riesgo de sangrado significativo: a) En pacientes con ACV previo, AIT o embolia sistémica, se puede considerar el uso de un anticoagulante parenteral como TP; b) En pacientes sin ACV previo, AIT o embolia sistémica, no es aconsejado(1).

- Pacientes con alto riesgo: se recomienda realizar TP salvo para los que presentaron hemorragia intracraneal reciente (tres meses) ${ }^{(1)}$.

\subsection{Terapia puente preprocedimiento para los que reciben inhibidores de vitamina $\mathrm{K}$}

1) Aunque la heparina no fraccionada (HNF) y la HBPM son las más comúnmente utilizadas, frente a antecedentes de trombocitopenia inducida por heparina, deben usarse anticoagulantes no heparínicos y ser seleccionados de acuerdo con la función renal y hepática.

2) Comenzar la terapia anticoagulante parenteral cuando el INR ya no es terapéutico.
3) Suspender la HNF $\geq 4$ horas antes del procedimiento; el efecto anticoagulante residual puede medirse con el tiempo de tromboplastina parcial activado (KPTT).

4) Interrumpir la HBPM al menos 24 horas antes del procedimiento; el efecto anticoagulante residual puede ser medido con el antifactor Xa específico de HBPM.

La decisión de utilizar HNF en lugar de HBPM depende de: 1) función renal (basada en $\mathrm{ClCr}$ ); 2) paciente hospitalizado o ambulatorio; 3) comodidad del paciente con las inyecciones.

Si ClCr es <30 ml/min, se prefiere HNF sobre HBPM. Sin embargo, la dosificación de HBPM puede ser ajustada para pacientes con un $\mathrm{ClCr}$ de 15 a $30 \mathrm{ml} / \mathrm{min}$.

\subsection{Interrupción y puente para pacientes en tratamiento con anticoagulantes orales directos}

Dada la vida media corta de los ACOD, suele no ser necesaria TP antes de los procedimientos. El reinicio de estos fármacos puede tener que retrasarse por el riesgo de sangrado posprocedimiento, la necesidad de procedimientos adicionales o la incapacidad del paciente para tolerar medicamentos orales ${ }^{(1)}$.

Salvo que la situación sea considerada de riesgo hemorrágico mínimo, en cuyo caso no se requiere la suspensión de ACOD (únicamente se omitirá la toma coincidente con el procedimiento), dicho tratamiento se suspenderá con una antelación que dependerá del riesgo hemorrágico del procedimiento y del tiempo necesario para que se produzca una pérdida del efecto anticoagulante (lo cual estará en relación, a su vez, con el aclaramiento de creatinina) $)^{(5)}$.

Los anticoagulantes parenterales (por ejemplo, HNF o HBPM) pueden ser necesarios entre los procedimientos o posprocedimiento cuando el riesgo trombótico es alto ${ }^{(1)}$.

La experiencia acerca de la seguridad y eficacia de la no interrupción de la anticoagulación con ACOD es limitada. En el estudio VENTURE-AF, los pacientes con FANV que iban a ablación no interrumpieron anticoagulación con rivaroxabán o AVK con el objetivo de valorar la incidencia de sangrado mayor y eventos TE. Los resultados mostraron que las tasas de sangrado mayor fueron bajas $(0,4 \%)$, al igual que los eventos TE $(0,8 \%)$. Una limitación fue el número pequeño de pacientes incluidos, por lo que es difícil extrapolar los resultados ${ }^{(11)}$. 
3. Cuándo y cómo debe reiniciarse la terapia anticoagulante para los pacientes que requieran interrupción transitoria Para el reinicio de la terapia con un AVKo ACOD es necesario asegurarse que la hemostasia se haya logrado y que se ha evaluado el riesgo hemorrágico del procedimiento y del paciente ${ }^{(1)}$.

\subsection{Reinicio de la terapia con inhibidores de vitamina $\mathrm{K}$}

En la mayoría de las situaciones un AVK se puede reiniciar en las primeras 24 horas posteriores en la dosis habitual.

El puente posprocedimiento con un agente parenteral puede considerarse en pacientes con riesgo moderado o alto de ACV, o evento TE y evitarse cuando se considera que hay riesgo de sangrado elevado por lo menos en las primeras 48 a 72 horas.

Cuando se reinicia el tratamiento con AVK, se requiere un cuidadoso monitoreo del INR durante el puente para mitigar el riesgo de sangrado.

La HBPM o HNF deben ser discontinuados cuando el INR esté en rango(1).

\subsection{Reinicio de la terapia con anticoagulantes orales directos}

Después de los procedimientos con bajo riesgo de sangrado es razonable reanudar el tratamiento con ACOD a dosis completa al día siguiente, mientras que en los de alto riesgo es razonable esperar 48 a 72 horas para retomarlo.

La dosis de ACOD se debe ajustar de acuerdo a la función renal posprocedimiento.

Por lo general, no se requiere puente con un agente parenteral.

Escenarios que requieren consideración especial para el reinicio de anticoagulantes orales directos

- Cuando un paciente tiene incapacidad para recibir medicamentos por vía oral durante períodos prolongados después de un procedimiento, se recomienda que la anticoagulación con un agente parenteral comience dentro de las primeras 24 horas después de un procedimiento con bajo riesgo de sangrado y dentro de las 48 a 72 horas después de un procedimiento con alto riesgo de sangrado ${ }^{(1)}$.

- Cuando se realiza anestesia neuroaxial se debe reiniciar el tratamiento con ACOD en las 24 horas posteriores o después de la extracción del catéter ${ }^{(1)}$.

\section{Conclusiones}

- Frente a cada paciente con FANV bajo tratamiento anticoagulante que será sometido a un procedimiento invasivo de coordinación, se debe evaluar el riesgo TE y de sangrado. Existen diferentes recomendaciones para la suspensión, según se trate de AVK o ACOD.

- En los pacientes de alto riesgo TE, la TP es necesaria, mientras que en los de bajo riesgo se puede prescindir de la misma. Existe discusión en los casos de riesgo moderado.

- Para reiniciar el tratamiento anticoagulante es necesario considerar el resultado de la hemostasis realizada, el riesgo hemorrágico del procedimiento y del paciente, así como el riesgo de TE.

\section{Bibliografía}

1. Doherty J, Gluckman T, Hucker W, Januzzi J, Ortel T, Saxonhouse S, et al. 2017 ACC expert consensus decision pathway for periprocedural management of anticoagulation in patients with nonvalvular atrial fibrillation: a report of the American College of Cardiology Clinical Expert Consensus Document Task Force. J Am Coll Cardiol 2017; 69(7): 87198.

2. Gómez A, Peixoto S, Azcúnaga M, Lluberas N, Silvera G, Álvarez $\mathbf{P}$, et al. Utilidad del score SAMe-TT2R2 en el control de la anticoagulación oral con warfarina en pacientes con fibrilación auricular no valvular. Rev Urug Cardiol 2016; 31(3):381-9.

3. Barrios V, Masjuan J. Use of direct oral anticoagulants in patients with nonvalvular atrial fibrillation according to clinical profile. Future Cardiol 2017; 13(1):49-64. doi: 10.2217/fca-2016-0061.

4. Morais J, De Caterina R. Stroke prevention in atrial fibrillation: a clinical perspective on trials of the novel oral anticoagulants. Cardiovasc Drugs Ther 2016; 30(2):201-14. doi: 10.1007/s10557- 0156632-3.

5. Sánchez Fuentes D, Budiño Sánchez M, López Sánchez M. Uso de la anticoagulación oral en el paciente sometido a un procedimiento invasivo. Rev Clin Esp 2017; 217(2):13-7. doi: 10.1016/j.rce. 2016. 06.003 .

6. Douketis J, Spyropoulos A, Kaatz S, Becker R, Caprini J, Dunn A, et al. Perioperative bridging anticoagulation in patients with atrial fibrillation. N Engl J Med 2015; 373(9):823-33. doi: 10.1056/ NEJMoa1501035.

7. Vivas D, Roldán I, Ferrandis R, Marín F, Roldán V, Tello-Montoliu A, et al. Manejo perioperatorio y periprocedimiento del tratamiento antitrombótico: documento de consenso de SEC, SEDAR, SEACV, SECTCV, AEC, SECPRE, SEPD, SEGO, SEHH, SETH, SEMERGEN, SEMFYC, SEMG, SEMICYUC, SEMI, SEMES, SEPAR, SENEC, SEO, SEPA, SERVEI, SECOT y AEU. Rev Esp Cardiol 2018; 71(7):553-64. doi: 10.1016/j.recesp.2018. 01.001. 
8. Birnie D, Healey J, Wells G, Verma A, Tang A, Krahn A, et al. Pacemaker or defibrillator surgery without interruption of anticoagulation. N Engl J Med 2013; 368(22):2084-93. doi: 10.1056/NEJMoa 1302946.

9. Siegal D, Yudin J, Kaatz S, Douketis J, Lim W, Spyropoulos A. Periprocedural heparin bridging in patients receiving vitamin $\mathrm{K}$ antagonists: systematic review and meta-analysis of bleeding and thromboembolic rates. Circulation 2012; 126(13):1630-9.

10. Steinberg B, Peterson E, Kim S, Thomas L,
Gersh B, Fonarow G, et al. Use and outcomes associated with bridging during anticoagulation interruptions in patients with atrial fibrillation: findings from the Outcomes Registry for Better Informed Treatment of Atrial Fibrillation (ORBIT-AF). Circulation 2015; 131(5):488-94.

11. Cappato R, Marchlinski F, Hohnloser S, Naccarelli G, Xiang J, Wilber D, et al. Uninterrupted rivaroxaban vs. uninterrupted vitamin $\mathrm{K}$ antagonists for catheter ablation in non-valvular atrial fibrillation. Eur Heart J 2015; 36(28):1805-11. doi: 10.1093/eurheartj/ehv177. 